\title{
FORMULACIÓN DE UN ANALISIS ESTRATEGICO SOBRE EL DESARROLLO EMPRESARIAL DE PAMPLONA UTILIZANDO LA HERRAMIENTA ESTRATEGICA MICMAC
}

\author{
Steven's Lopesierra Pinto* \\ Luis Manuel Palomino Méndez **
}

\footnotetext{
* Estudiante prospectiva II semestre 2008. E-mail: Stevens.lopesierra@unipamplona.edu.co

** Docente facultad ciencias económicas y empresariales-Universidad de Pamplona. Pamplona Colombia. E-mail: luismanuel@unipamplona.edu.co
} 


\section{FORMULACIÓN DE UN ANALISIS ESTRATEGICO SOBRE EL DESARROLLO EMPRESARIAL DE PAMPLONA UTILIZANDO LA HERRAMIENTA ESTRATEGICA MICMAC}

\section{RESUMEN}

El análisis estratégico del desarrollo empresarial de pamplona es un tema de actualidad ante las circunstancias que se viven en términos del estancamiento o progreso moderado del sector empresarial de la ciudad mitrada.

La formulación del análisis estratégico utilizando la herramienta del MICMAC, representa la visión prospectiva de los posibles escenarios que para el año 2020, partiendo en la definición de variables que en forma directa o indirecta se determinan en esta época.

La ubicación geopolítica acompañada de fortalezas como el talento humano, los recursos minerales, agrícolas entre otros; fijan pautas que incentivan a los administradores a vislumbrar el futuro dentro de un entorno positivo, en donde los diferentes actores del tejido social participen y se beneficien enmarcados dentro de un plan de desarrollo posible.

Palabras Claves: Desarrollo empresarial, análisis estratégico, MICMAC.

\begin{abstract}
The analysis of strategic business development in Pamplona is a topical issue in the circumstances being experienced in terms of stagnation or moderate progress of the business sector of the city Mitra.
\end{abstract}

The formulation of the strategic analysis using the tool MICMAC, is the prospective view of the possible scenarios for the year 2020, based on the definition of variables that directly or indirectly determine at this time.

The geopolitical location with strengths as human talent, mineral resources, including agricultural, setting guidelines that encourage managers to envision the future in a positive environment, where different social actors to participate in and benefit within the framework of a development plan possible.

Key Words: Business development, strategic analysis, MICMAC.

Face issN 1794-9920

Recepción: Enero de 2011

Revisión: Junio de2011

Aceptación: Junio de 2011 


\section{INTRODUCCIÓN}

La iniciativa del artículo tiene como finalidad la de aportar desde el punto de vista de los estudiantes da la asignatura prospectiva del programa de administración de empresas de generar mediante la herramienta MICMAC una planeación prospectiva utilizando el escenario Desarrollo empresarial - Pamplona 2020, para llevar a cabo este análisis, se formularon 30 variables que tienen un impacto directo en los diferentes sectores económicos presentes en la ciudad.

El mencionado programa, desarrolla dentro de su programación la presentación de graficas y planos en donde se observan la relación de las variables inmersas en el proceso permitiendo encontrar relaciones entre las variables que facilitan $u$ obstaculizan el tema del estudio.

\section{PRESENTACIÓN DE LAS VARIABLES}

\section{Descripción de las variables.}

A continuación se enumeran y definen las variables que se tomaron en cuanta para el análisis prospectivo.

\section{Deserción Estudiantil (DES EST)}

\section{Descripción:}

La deserción estudiantil se puede definir como el proceso de abandono, voluntario o forzoso, del programa académico en el que se matriculó un estudiante universitario. Este fenómeno obedece a causas, internas y externas, que involucran factores personales, familiares, socioeconómicos, culturales e institucionales. Se relaciona también con aspectos como el ausentismo, el retiro forzoso, el cual es recurrente, conduce al abandono definitivo de los estudios.

\section{Universidad De Pamplona (UP)}

\section{Descripción:}

La Universidad de Pamplona es la principal institución académica del departamento de Norte de Santander y una de las universidades públicas más prestigiosas de Colombia. Fue fundada el 23 de noviembre de 1960, tiene su sede principal en el municipio Pamplona y es una de las 500 empresas más grandes del país. Cuenta con 7 vicerrectorías, se divide académicamente en 9 facultades y su máximo organismo de gobierno es la Secretaría General. El actual rector(a) es la Dra. Esperanza Paredes De Estévez 


\section{Cultura (CULT)}

\section{Descripción:}

La cultura es el conjunto de todas las formas y expresiones de una sociedad determinada. Como tal incluye costumbres, prácticas, códigos, normas y reglas de la manera de ser, vestimenta, religión, rituales, normas de comportamiento y sistemas de creencias. Desde otro punto de vista se puede decir que la cultura es toda la información y habilidades que posee el ser humano y algunos animales. El concepto de cultura es fundamental para las disciplinas que se encargan del estudio de la sociedad.

\section{Contrabando (CONT)}

\section{Descripción:}

Contrabando es la entrada o la salida y venta clandestina de mercancías prohibidas o sometidas a derechos en los que se defrauda a las autoridades locales.

\section{Salida De Capital (SALCAP) \\ Descripción:}

Salida de Capital. Salida de recursos financieros fuera de la región, estimulada por mayor rendimiento o seguridad ofrecida en los mercados nacionales o internacionales.

\section{Reinversión (RE) \\ Descripción:}

Reinversión. Inversión de los beneficios obtenidos de una inversión previa en el mismo negocio.

\section{Desarrollo Endógeno (DES ENDG) \\ Descripción:}

Desarrollo endógeno es un modelo de desarrollo que busca potenciar las capacidades internas de una región o comunidad local; de modo que puedan ser utilizadas para fortalecer la sociedad y su economía de adentro hacia afuera, para que sea sustentable y sostenible en el tiempo. Es importante señalar que en el desarrollo endógeno el aspecto económico es importante, pero no lo es más que el desarrollo integral del colectivo y del individuo: en el ámbito moral, cultural, social, político, y tecnológico.

\section{Creación De Una Zona Franca (ZF) Descripción:}

Las Zonas Francas son áreas geográficas específicas cuyo objetivo es la industrialización con fines de exportación. 


\section{Creación De Capital Semilla En El Municipio Para El Fomento Empresarial (CAP SEMI) \\ Descripción:}

Se entiende por capital de semilla a la cantidad de dinero necesaria para implementar una empresa y financiar actividades claves en el proceso de iniciación y puesta en marcha. El capital es aportado por terceros.

\section{Usualmente este capital se destina a:}

- Compra de activos y capital de trabajo.

- Desarrollar prototipos, lanzar un producto o servicio al mercado.

- Proteger una innovación, propiedad intelectual (registro de marcas, patentes).

- Constitución y puesta en marcha de la empresa.

\section{Tradición Empresarial (TRA EMP)}

\section{Descripción:}

Forma de conducta propia de un grupo humano, que tiene origen antiguo y se transmite de generación en generación.

\section{Conformismo Económico (CON ECO) \\ Descripción:}

El conformismo es una de las causas de la pobreza. El cambio crea prosperidad.

¿Por qué la gente se conforma con lo que tiene y vive año tras año, sin prosperar? Mucha gente se jubila y lo único que les ampara es el cheque de la jubilación; otros, ni siquiera eso. Nuestros padres, tíos, parientes llegan a viejos sin haber cumplido sus anhelos.

El motivo fue, que se conformaron con lo que tenían y no empezaron una nueva aventura en su vida, por el temor al cambio y a lo desconocido.

\section{Rueda De Negocios (R NEG) Descripción:}

La Rueda de Negocios es una actividad para conectar a expositores y visitantes a través de una presentación comercial, de aproximadamente 10 minutos, en la que el franquiciador cuenta su historia, la modalidad de negocio y las condiciones para entregar a posibles inversionistas su franquicia.

\section{Tecnificación Del Campo (TEC CAM) \\ Descripción:}

Tecnificación del campo permite mejorar las condiciones de trabajo y elevar los ingresos 


\section{Capacitación Microempresarial (CAP EMP) Descripción:}

Capacitación, o desarrollo de personal, es toda actividad realizada en una organización, respondiendo a sus necesidades, que busca mejorar la actitud, conocimiento, habilidades o conductas de su personal.

La necesidad de capacitación surge cuando hay diferencia entre lo que una persona debería saber para desempeñar una tarea, y lo que sabe realmente.

Estas diferencias suelen ser descubiertas al hacer evaluaciones de desempeño, o descripciones de perfil de puesto.

\section{Infraestructura Vial (INF VIAL) Descripción:}

La infraestructura vial es uno de los pilares de crecimiento y desarrollo sostenible del país. Pese a su importancia económica y social, presenta altos niveles de atrasos. Se identifica la falta e inadecuada planeación como una de las razones del rezago en el sector vial.

\section{Comercio Informal (COM INF) Descripción:}

El comercio Informal es un fenómeno que se da en casi todas las ciudades del mundo, Se caracteriza principalmente porque quienes se dedican a esta actividad, no cuentan con un local o espacio para la realización de sus labores, ni están sujetos a todos los procesos de formalización social impartidos en la legislación económica nacional.

\section{Inversión Del Gobierno (INV GOB) Descripción:}

Es el acto mediante el cual se cambia una satisfacción inmediata y cierta, por una esperanza, que se adquirirá a cambio de una adecuada compensación y de la que soporte el bien en que se invierte. La inversión se hace con la esperanza de una recompensa en el futuro. La inversión se refiere al empleo de un capital en algún tipo de actividad o negocio con el objetivo de incrementarlo. Dicho de otra manera, consiste en posponer al futuro un posible consumo.

\section{Ubicación Geopolítica (UB GEOP) Descripción:}

El conocimiento geopolítico de un Estado se logra mediante el estudio de las condiciones en que éste desarrolla su espacio vital. Para tal efecto se hace necesario considerar los elementos básicos de la Geopolítica como son: la Realidad Nacional, el Poder Nacional, el Potencial Nacional y la Política Nacional. 


\section{Cadena Productiva (CAD PROD) Descripción:}

Las cadenas productivas como el conjunto de actividades que se articulan técnica y económicamente desde el inicio de la producción y elaboración de un producto agropecuario, hasta su comercialización final. La Cadena puede ser conformada de común acuerdo, a nivel nacional, a nivel de una zona o región productora, por los productores, empresarios, gremios y organizaciones más representativos tanto de la producción agrícola, pecuaria, forestal, acuícola, pesquera, como de la transformación y la comercialización.

\section{Desarrollo Del Ecoturismo (DES ECO)}

Descripción:

El ecoturismo es, cada vez más, parte integral del turismo y, como tal, puede surtir el impacto socio-económico nacional que es propio a ese sector. El ecoturismo incrementa la producción de bienes y servicios, y ayuda a generar empleos e ingresos.

El ecoturismo tiene un impacto económico como sector particular y como parte de la actividad turística en general. Por su naturaleza, el ecoturismo estimula, a la vez que da cabida al nacimiento de micro, pequeños y medianos negocios, tanto rurales como urbanos.

\section{Inestabilidad Económica (INS ECO) \\ Descripción:}

La inestabilidad económica del país, la desigualdad para competir y prácticas comerciales en el mercado interno no permiten que las micro, pequeñas y medianas empresas (MPyMES) crezcan.

\section{Implementación De La Cátedra Empresarial Desde La Primaria (IMP CAT) Descripción:}

La cátedra empresarial tendiente precisamente a transmitir conocimiento, formar actitudes favorables en los alumnos sobre el emprendimiento, la innovación, la creatividad y por supuesto, desarrollar las competencias para generar empresa.

\section{Competencias Laborales (COM LAB) Descripción:}

En el creciente y diverso conjunto de libros, artículos y materiales de diferente tipo que se viene acumulando respecto al tema, existen múltiples caracterizaciones y definiciones (que provocan otro de los riesgos: el de los malentendidos semánticos). 
Partiendo de una definición bastante aceptada, podemos entender a las competencias como el conjunto de conocimientos, habilidades y actitudes verificables, que se aplican en el desempeño de una función productiva.

\section{Formación Integral Del Capital Humano (FOR CP HUM) Descripción:}

A formación de capital humano es hacer que el conocimiento esté dispuesto a la cualificación de los recursos humanos y sociales, que giren alrededor de un solo objetivo, que permita beneficiar a la población y al desarrollo colectivo de la subregión.

Entendida la formación de capital humano como la preparación adecuada de amplios sectores de la comunidad, productores rurales, agentes individuales y actores sociales estratégicos en la perspectiva del desarrollo rural agroindustrial sostenible.

\section{Implementación De Investigación $Y$ Desarrollo Económico En Pamplona (IMP ECO) \\ Descripción:}

En el contexto de comercio, "investigación y desarrollo" se suele referir a actividades de largo recorrido orientadas al futuro en tecnología o ciencia copiando la investigación científica que no se vincula tanto a la búsqueda de beneficios.

\section{Adaptación Al Entorno (AD ENT) Descripción:}

La adaptación al entorno representa uno de los principales elementos que puede paliar o mitigar el grado de dependencia de la comunidad ante diferentes cambios.

\section{Responsabilidad Social Empresarial (RSE) Descripción:}

La Responsabilidad Social es la capacidad de respuesta que tiene una empresa o una entidad, frente a los efectos e implicaciones de sus acciones sobre los diferentes grupos con los que se relaciona (stakeholders o grupos de interés). De esta forma las empresas son socialmente responsables cuando las actividades que realiza se orientan a la satisfacción de las necesidades y expectativas de sus miembros, de la sociedad y de quienes se benefician de su actividad comercial.

\section{Exportaciones De Productos (X PRO) Descripción:}

La salida de mercancías del territorio Aduanero Nacional a una Zona Franca Industrial de bienes y servicios. 


\section{Cumplimiento De La Política Laboral (POL LAB) Descripción:}

La política laboral en general incluye las leyes y normas vigentes en tu país que afecten al ámbito laboral. Estarán influidas además por la actuación de las asociaciones empresariales y los sindicatos.

\section{Observatorio Económico (OBS ECO) Descripción:}

Organismo de seguimiento al comportamiento de los principales indicadores de la economía de la ciudad.

\section{Figura 1. Matriz de Influencias Directas (MID)}

Esta matriz describe cuantitativamente la relación existente entre las diferentes variables formuladas para el análisis prospectivo.

\begin{tabular}{|c|c|c|c|c|c|c|c|c|c|c|c|c|c|c|c|}
\hline & $\begin{array}{l}\vec{\sigma} \\
\ddot{2} \\
0 \\
0 \\
\vdots \\
\text { ż }\end{array}$ & $\begin{array}{c}\vec{v} \\
\cdots \\
\bar{z} \\
\bar{\Omega} \\
0 \\
0 \\
0\end{array}$ & $\begin{array}{c}\overrightarrow{0} \\
\ddot{c} \\
\widetilde{\omega} \\
\Omega \\
\text { m. } \\
0 \\
0\end{array}$ & \begin{tabular}{|l|}
$\overrightarrow{0}$ \\
$\ddot{0}$ \\
$\rho$ \\
0 \\
0 \\
0 \\
0 \\
0
\end{tabular} & 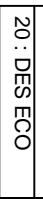 & $\begin{array}{c}N \\
\vec{A} \\
\bar{\Sigma} \\
\text { क } \\
\text { m } \\
\text { О }\end{array}$ & 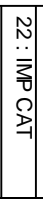 & 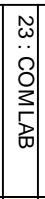 & 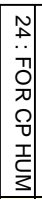 & 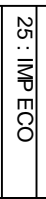 & 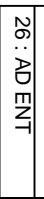 & 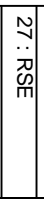 & $\begin{array}{l}N \\
\infty \\
\ddot{x} \\
0 \\
0 \\
0\end{array}$ & 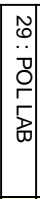 & 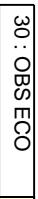 \\
\hline $1:$ deserción estudiantil & 2 & 3 & 2 & 1 & 2 & 3 & 2 & 2 & 2 & \begin{tabular}{|l|}
3 \\
\end{tabular} & 2 & 0 & 0 & 0 & 0 \\
\hline 2: universidad de pamplona & 2 & 3 & 3 & 3 & 3 & 3 & 3 & 3 & 3 & 1 & 2 & 1 & 2 & 3 & 3 \\
\hline 3 : cultura & 3 & 1 & 3 & 3 & 3 & 2 & 0 & 3 & 3 & 3 & 2 & 3 & 3 & 3 & 2 \\
\hline 4 : contrabando & 3 & 3 & 3 & 3 & 1 & 3 & 0 & 1 & 2 & 3 & 1 & 3 & 3 & 2 & 2 \\
\hline 5: salida de capital & 2 & 3 & 2 & 3 & 1 & 3 & 1 & 0 & 2 & 2 & 0 & 2 & 2 & 2 & 3 \\
\hline 6: reinverción & 3 & 3 & 3 & 3 & 3 & 1 & 2 & 2 & 2 & 2 & 2 & 1 & 2 & 1 & 2 \\
\hline 7: desarrollo en dogeno & 3 & 3 & 3 & 3 & 3 & 1 & 0 & 0 & 3 & 3 & 3 & 3 & 2 & 2 & 3 \\
\hline 8: creación de una zona franca & 1 & 1 & 2 & 2 & 2 & 2 & 1 & 1 & 2 & 2 & 2 & 2 & 3 & 2 & 2 \\
\hline 9: creacion de capital semilla en el municipio para el fomento empresarial & 2 & 1 & 1 & 2 & 3 & 2 & 1 & 2 & 2 & 2 & 2 & 2 & 1 & 2 & 2 \\
\hline 10 : tradición empresarial & 2 & 2 & 2 & 3 & 3 & 3 & 1 & 2 & 2 & 2 & 2 & 1 & 2 & 3 & 1 \\
\hline 11 : conformismo economico & 2 & 2 & 3 & 3 & 2 & 3 & 0 & 2 & 3 & 3 & 2 & 2 & 1 & 2 & 2 \\
\hline 12 : rueda de negocios & 2 & 3 & 3 & 3 & 3 & 3 & 0 & 1 & 2 & 2 & 1 & 3 & 2 & 2 & 3 \\
\hline 13 : tecnificacion del campo & 2 & 3 & 3 & 3 & 3 & 0 & 2 & 3 & 2 & 3 & 1 & 2 & 3 & 1 & 3 \\
\hline 14 : capacitación microempresarial & 2 & 2 & 2 & 3 & 3 & 3 & 1 & 0 & 1 & 2 & 2 & 2 & 3 & 2 & 2 \\
\hline 15 : infraestructura vial & 1 & 3 & 3 & 3 & 3 & 2 & 1 & 2 & 0 & 2 & 1 & 3 & 3 & 3 & 2 \\
\hline 16 : comercio informal & 0 & 2 & 2 & 2 & 2 & 3 & 0 & 1 & 1 & 3 & 1 & 3 & 2 & 2 & 2 \\
\hline 17 :inverción del gobierno & 3 & 0 & 3 & 3 & 3 & 2 & 1 & 0 & 2 & 2 & 2 & 3 & 2 & 3 & 3 \\
\hline 18: ubicación geopolitica & 2 & 2 & 0 & 3 & 3 & 3 & 0 & 3 & 2 & 3 & 2 & 2 & 1 & 2 & 2 \\
\hline 19 : cadena productiva & 2 & 3 & 3 & 0 & 3 & 2 & 2 & 0 & 1 & 2 & 2 & 2 & 2 & 3 & 2 \\
\hline 20 : desarrollo del ecoturismo & 3 & 3 & 3 & 3 & 0 & 2 & 2 & 1 & 0 & 3 & 2 & 1 & 1 & 2 & 2 \\
\hline 21 : inestabilidad economica & 1 & 3 & 3 & 3 & 3 & 0 & 1 & 1 & 2 & 2 & 2 & 2 & 2 & 2 & 3 \\
\hline 22 : implementacion de la catedra empresarial desde la primaria & 0 & 2 & 2 & 1 & 1 & 1 & 0 & 1 & 3 & 2 & 2 & 2 & 2 & 1 & 2 \\
\hline 23: competencias laborales & 2 & 1 & 2 & 2 & 2 & 3 & 1 & 0 & 2 & 2 & 3 & 1 & 3 & 3 & 1 \\
\hline 24 : formacion integral del capital humano & 2 & 1 & 2 & 2 & 2 & 3 & 1 & 2 & 0 & 2 & 3 & 2 & 3 & 3 & 3 \\
\hline 25 : implementacion de investigacion y desarrollo economico en pamplona & 3 & 3 & 3 & 3 & 3 & 1 & 1 & 1 & 2 & 0 & 3 & 2 & 3 & 3 & 2 \\
\hline 26: adaptacion al entorno & 2 & 1 & 2 & 2 & 2 & 1 & 1 & 1 & 3 & 2 & 0 & 1 & 2 & 2 & 3 \\
\hline 27 : responsabilidad social empresarial & 2 & 3 & 3 & 3 & 3 & 1 & 1 & 0 & 1 & 3 & 2 & 0 & 3 & 3 & 2 \\
\hline 28: exportaciones de productos & 0 & 2 & 2 & 3 & 3 & 1 & 0 & 2 & 0 & 2 & 2 & 1 & 0 & 2 & 2 \\
\hline 29: cumplimiento de la politica laboral & 1 & 1 & 3 & 2 & 1 & 1 & 0 & 0 & 0 & 1 & 2 & 2 & 2 & 0 & 2 \\
\hline 30 : observatorio economico & 2 & 2 & 2 & 3 & 1 & 1 & 0 & 1 & 2 & 2 & 2 & 2 & 3 & 2 & 0 \\
\hline
\end{tabular}

Como se observa en la figura 1, el software MICMAC se alimentó con las variables que previamente fueron definidas, los resultados que se obtienen a partir de esta evaluación de esta matriz de nivel 1, reflejan el tipo de influencia entre las variables 
de acuerdo al impacto del entorno y con proyección a futuro en el caso de estudio, para llevar a cabo este proceso de calificación a continuación se relacionan el siguiente parámetro de valoración.

Las influencias se puntúan de 0 a 3 , con la posibilidad de señalar las influencias potenciales:

$0:$ Sin influencia

1: Débil

2: Media

3: Fuerte

P: Potencial

Como se observa no existe una gran diferencia en la calificación entre las variables, los que nos indica que el nivel de relación es muy homogéneo concluyéndose que no hay un sector que haga la diferencia, por muy alto o muy bajo. Sino que existe una media de impacto de cada uno de los sectores presentes con respecto al DESARROLLO EMPRESARIAL de la ciudad de pamplona.

Figura 2. Resultado De La Alimentación De La Primera Matriz (MID) Plano de influencias / dependencias directas

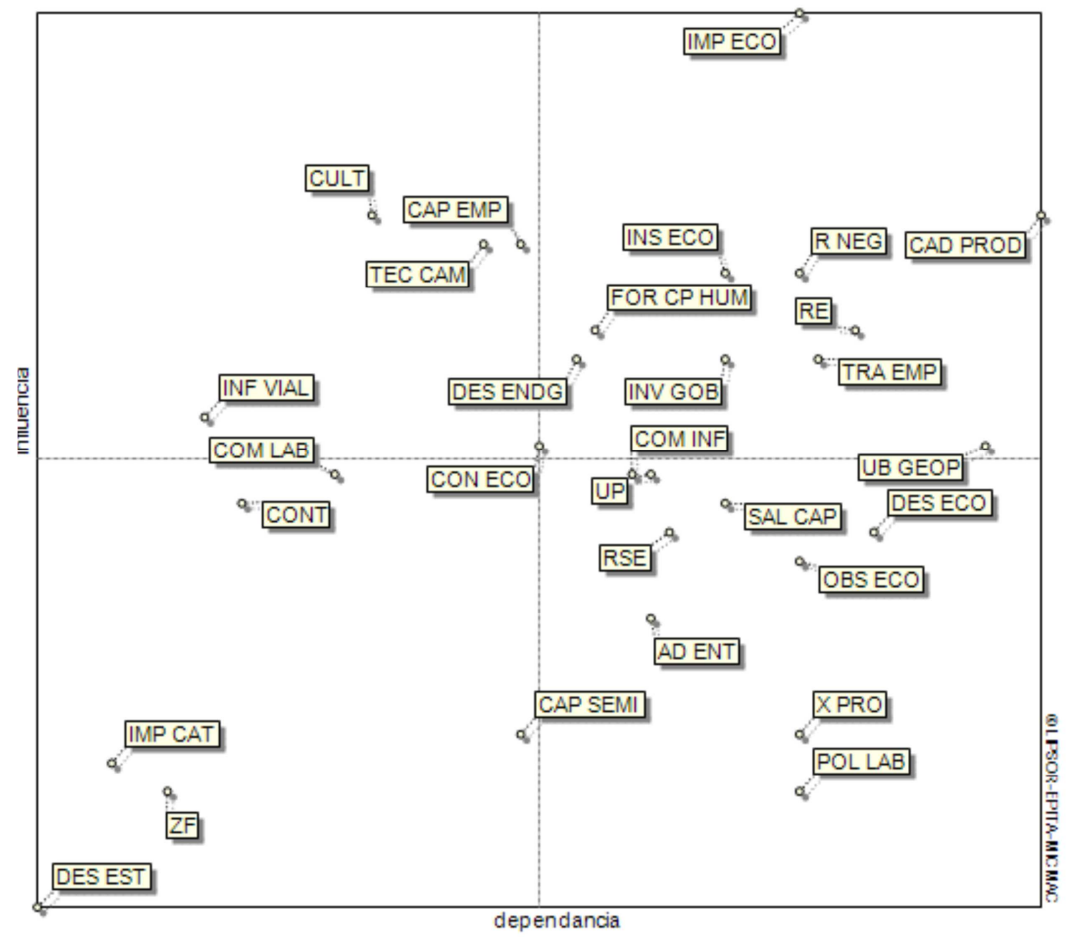


La figura 2, En la gráfica del plano se puede observar la distribución de la variables dentro de la conformación que de acuerdo al software se establece, en el cuadrante de enlace que es donde se ubican la variables claves, El Desarrollo Endógeno, La Inversión Del Gobierno Y La Formación Integral Del Capital Humano son tomadas por el proceso de planeación.

Que las mencionadas variables estén dentro de este cuadrante demuestra que para obtener el desarrollo empresarial de pamplona es necesario que se cumplan estas tres (3) variables, si bien es cierto esto es positivo hay que tener en cuenta que la variables claves son dependientes haciéndose necesario establecer políticas que en un escenario futuro se observen con la creación de unidades productivas basados en las variables que fueron evaluadas y relacionadas en el cuadrante de enlace, en el área de las variables de exclusión se ubican: Deserción Estudiantil, Creación De Una Zona Franca Y Implementación De La Cátedra Empresarial Desde La Primaria.

Estas variables se denominan Autónomas por qué no necesitan ni ser influenciadas ni generan dependencias sobre otras, es decir se consideran desconectadas del sistemas. Si analizamos la variable ubicada en este cuadrante se puede analizar que la zona franca no correspondería a esta definición porque de su implementación depende de una política de inversión empresarial y su origen no es la resultante de una iniciativa vaga y desconectada del proceso las dos (2) variables restantes si corresponden a su definición. En el cuadrante de entrada: La Cultura, La Infraestructura Vial, La Capacitación De Microempresarios Y La Tecnificación Del Campo. Se consideran vitales para que se lleve a cabo el desarrollo empresarial de pamplona, este tipo de variables son influyentes y por ende su dependencia es casi nula. Y afectan de manera directa a las variables del cuadrante de enlace.

Figura 3. Grafico De Influencias Directas

Este gráfico se determina a partir de la matriz de influencias directas MID.

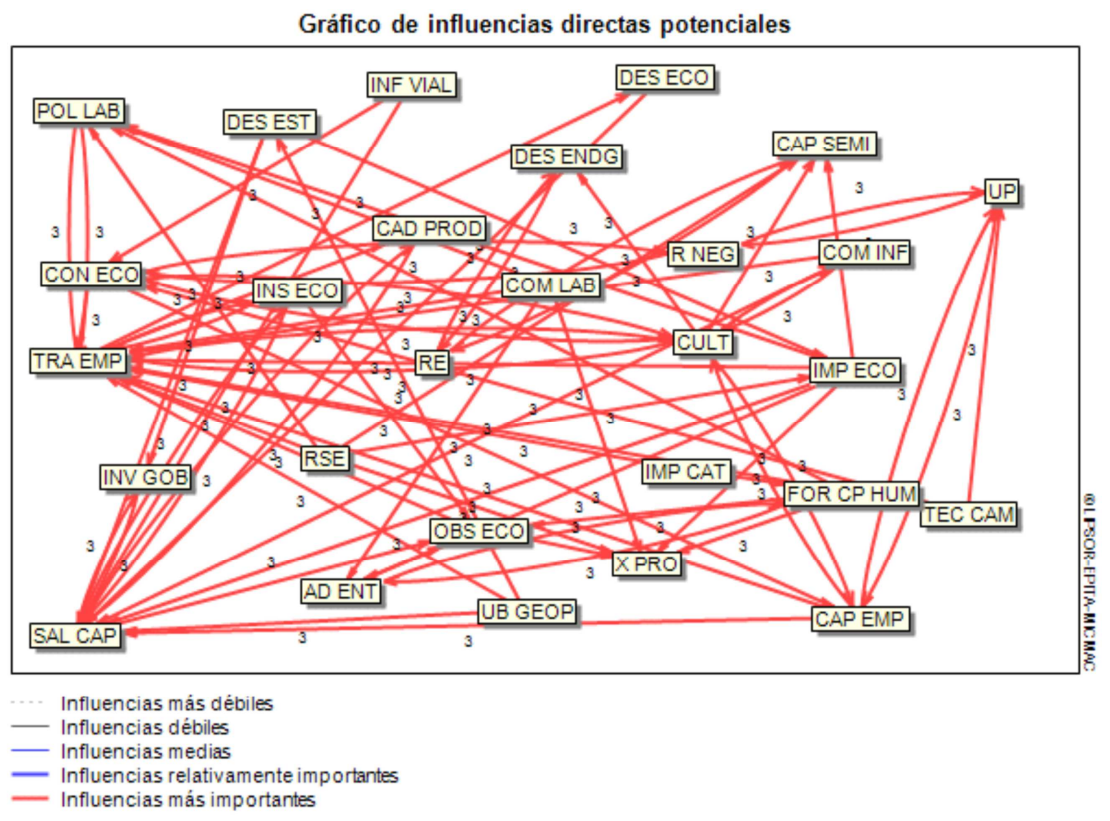


FORMULACIÓN DE UN ANALISIS ESTRATEGICO SOBRE EL DESARROLLO EMPRESARIAL DE PAMPLONA UTILIZANDO LA HERRAMIENTA ESTRATEGICA

MICMAC

La grafica muestra, la relación existente entre las variables analizadas dentro de las características de la dependencia y la influencia siendo las variables que más influyen:

- La tradición empresarial

- La capacitación microempresarial

- La adaptación al entorno

Y las que más presentan dependencia son:

- Inestabilidad económica

- Conformismo económico

- Salida de capital

En ausencia de criterios matemáticamente establecidos, se elige apoyarse en un número de permutaciones necesarios en cada interacción para clasificar, la influencia y la dependencia, del conjunto de variables.

Figura 4. Matriz de Influencias Indirectas Potenciales (MIIP)

\section{Gráfico de influencias indirectas potenciales}

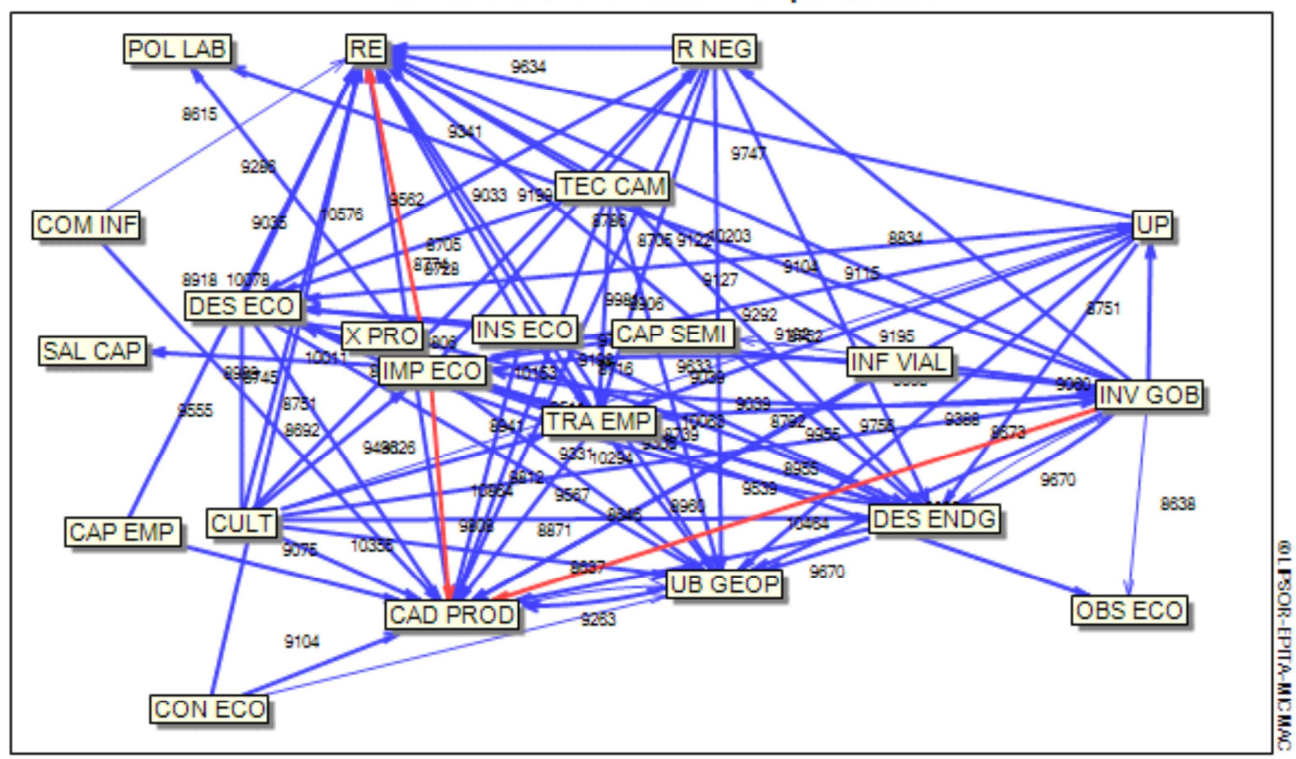

\footnotetext{
- Influencias más débiles

- Influencias débiles

- Influencias medias

- Influencias relativamente importantes

- Influencias más importantes
}

En esta figura se puede apreciar que las variables (universidad de pamplona, cultura y tradición empresarial) son las que más influyen relativamente; en influencia relativa potencial. 
En la figura 4, también se puede contemplar cuales son las variables con influencias más importantes:

- Inversión del gobierno

- Reinversión

- Exportación de productos

- Cadena productiva

\section{CONCLUSIONES}

Los resultados obtenidos a través del desarrollo del proceso del software MICMAC nos indican cuales son las variables que más influyen dentro del contexto del escenario propuesto:

- Inversión del gobierno

- Reinversión

- Exportación de productos

- Cadena productiva

Estos conllevan de forma directa al desarrollo empresarial de pamplona mediante un proceso de 11 años, generando una evolución en el desarrollo económico y empresarial de pamplona.

Todo esto fue realizado contemplando que la prospectiva es un modelo de planeación que estudia el futuro para comprenderlo y poderlo influir. Aunque de hecho es, paradójicamente, una ciencia sin objeto que se mueve entre la necesidad de predecir lo que puede ocurrir y el deseo de inventar el mejor futuro posible. 